\title{
Estabilidad de la austenita retenida bajo condiciones de deformación en aceros multifásicos con bainita nanoestructurada
}

\section{Stability of retained austenite under strain conditions on multiphase steels with nanostructured bainite}

\author{
Tania Loaiza Uribe ${ }^{1}$, Paula Andrea Pérez Espitia ${ }^{1}$, \\ Claudia Patricia Serna Giraldo ${ }^{1}$, Ricardo Emilio Aristizábal Sierra ${ }^{1}$
}

\footnotetext{
${ }^{1}$ Grupo de Investigaciones Pirometalúrgicas y de Materiales - GIPIMME, Departamento de Ingeniería Metalúrgica y de Materiales, Facultad de Ingeniería, Universidad de Antioquia, Medellín, Medellín,Colombia. e-mail: tania.loaiza31@gmail.com, pandrea.perez@udea.edu.co, claudia.serna@udea.edu.co, ricardo.aristizabal@udea.edu.co
}

\section{RESUMEN}

Se fabricó un acero de composición $0.25 \mathrm{C}, 1.9 \mathrm{Si}, 0.63 \mathrm{Mn}, 1.5 \mathrm{Cr}$ utilizando un horno de inducción. La aleación as-cast se homogenizó y se determinaron las temperaturas de inicio $\left(\mathrm{A}_{\mathrm{C} 1}\right)$ y finalización $\left(\mathrm{A}_{\mathrm{C} 3}\right)$ de la austenita durante el calentamiento mediante dilatometría. Se seleccionaron dos temperaturas de austenización intercrítica, que se utilizaron para realizar tratamientos térmicos de austenización parcial seguidos de tratamiento isotérmico en sales fundidas. Se caracterizaron los aceros tratados térmicamente con microscopia óptica, microscopía electrónica de barrido y difracción de rayos x. Las microestructuras obtenidas fueron multifásicas con ferrita proeutectoide, martensita, bainita y austenita retenida de tamaño nanométrico. Los aceros obtenidos fueron deformados plásticamente por laminación en frio para evaluar la estabilidad de la austenita bajo condiciones de deformación. Se encontró que la fracción de austenita retenida aumentó y su estabilidad bajo condiciones de deformación disminuyó, al disminuir la temperatura de austenización intercrítica. La combinación de austenización intercrítica seguida de austemperado a baja temperatura de una aleación con la composición química adecuada, permitió obtener aceros que combinan la microestructura de los aceros TRIP y NANOBAIN.

Palabras clave: aceros multifásicos, austenita retenida, transformación inducida por deformación plástica (TRIP)

\section{ABSTRACT}

A steel with nominal composition $0.25 \mathrm{C}, 1.9 \mathrm{Si}, 0.63 \mathrm{Mn}, 1.5 \mathrm{Cr}$ was manufactured using an induction furnace. The as-cast alloy was homogenized and the temperatures of austenite start $\left(\mathrm{A}_{\mathrm{C} 1}\right)$ and finish $\left(\mathrm{A}_{\mathrm{C} 3}\right)$ during heating were determined by dilatometric analyses. Two intercritical austenitization temperatures were selected, which were used to perform partial austenization followed by isothermal treatment in a salth bath. The heat treated steel was characterized by optical microscopy, scanning electron microscopy, and x-ray diffraction. The microstructures contained proeutectoid ferrite, martensite, bainite and nanometric retained austenite. These steels were subjected to plastic deformation by cold rolling to evaluate the stability of the retained austenite under strain. It was found that the fraction of retained austenite increased and its stability under deformation conditions decreased as the intercritical austenitized temperature diminished. The combination of intercritical austenization followed by low temperature austempering on an alloy with suitable chemical composition allowed to obtain steels that combine the microstructures of TRIP and Nanobain steels.

Keywords: multiphase steel, retained austenite, transformation induced plasticity (TRIP).

\section{INTRODUCCIÓN}

Los aceros TRIP (transformación inducida por deformación plástica por sus siglas en inglés) se caracterizan por poseer una buena combinación de propiedades mecánicas como alta resistencia última a la tensión, moderado límite de cedencia, alta ductilidad y elevado índice de endurecimiento por deformación. Sus propiedades 
mecánicas típicas incluyen resistencias últimas a la tracción de $1 \mathrm{GPa}$ y elongaciones que alcanzan el $40 \%$ [1]. Esta combinación de propiedades se debe a su microestructura formada por una matriz dúctil de ferrita con islas de bainita, martensita y austenita retenida [2]. Los aceros TRIP se utilizan en aplicaciones de la industria automovilística en piezas estructurales y de seguridad [3],[4]. Su fabricación incluye un ciclo termomecánico con austenización intercrítica y posterior austemperado [5],[6].

Por otro lado, se encuentran también los aceros bainíticos nanoestructurados conocidos como NANOBAIN, que representan una nueva generación de aceros con altas resistencias últimas a la tensión (1.7-2.2 GPa) y ductilidades entre 5\% y $30 \%$. Los aceros Nanobain son aceros nanocristalinos que se obtienen mediante tratamiento isotérmico a temperaturas bajas $\left(150^{\circ} \mathrm{C}-350^{\circ} \mathrm{C}\right)$, a las cuales la difusión del hierro es inconcebible durante la transformación. De esta forma, se obtiene una microestructura constituida por placas de ferrita bainítica y austenita retenida libre de carburos de tamaño nanométrico [7].

La presencia de austenita retenida en ambos materiales tiene una incidencia importante en su comportamiento mecánico. Bajo condiciones de carga la austenita puede transformarse a martensita por efecto de la deformación plástica, en un fenómeno conocido como TRIP. Esta transformación es favorable cuando ocurre a deformaciones plásticas elevadas ya que aumenta la deformación uniforme, la resistencia mecánica, la elongación y el coeficiente de endurecimiento por deformación [8], [9]. Se sabe que la composición química, en especial la concentración de carbono, el tamaño y la morfología de la austenita determinan su estabilidad frente a la deformación plástica. Por ejemplo, cuando la concentración de carbono es menor a $0.06 \%$ la austenita se transforma a martensita en las primeras etapas de la deformación, sin contribuir al aumento de la ductilidad, por otro lado cuando la concentración de carbono en la austenita es mayor a $1.8 \%$ en peso no ocurre transformación y por lo tanto tampoco hay una contribución al aumento de la elongación [10]. Se ha encontrado también que unidades de austenita con tamaño mayores a $1 \mu \mathrm{m}$ se transforman a martensita a bajas deformaciones, mientras las unidades de austenita de tamaño submicrométrico son más estables. Finalmente, se ha comprobado que la austenita en bloque es menos estable que las partículas de austenita que se encuentran entre láminas de ferrita bainítica [1], [11], [12].

En teoría, al combinar una austenización intercrítica seguida de austemperado a baja temperatura de una aleación con la composición química adecuada, se pueden obtener aceros que combinen las ventajas de los aceros TRIP y NANOBAIN, con microestructuras multifásicas compuestas por ferrita proeutectoide y bainita nanoestructurada con austenita retenida. En un material con esta microestructura, estudiar la estabilidad de la austenita retenida bajo condiciones de deformación se hace importante, ya que esta transformación como se mencionó anteriormente puede tener una marcada incidencia en las propiedades mecánicas. En este artículo se explora la posibilidad de obtener un acero de estas características, además se estudió la transformación de la austenita retenida bajo condiciones de deformación y el efecto de la temperatura de austenización intercrítica.

\section{MATERIALES Y MÉTODOS}

Se fabricaron $20 \mathrm{~kg}$ de acero en un horno de inducción (50KW- marca Induction Technology Corporation), utilizando como materiales de carga chatarra de acero de bajo carbono, grafito, ferrocromo, ferrosilicio y ferromanganeso. El material se vació en moldes de arena en verde y se obtuvieron probetas en Y con un espesor de una pulgada. La composición química se determinó mediante espectrometría de emisión óptica en un espectrómetro marca Bruker Q8 Magellan dando como resultado C: $0.25 \%$, Si: $1.9 \%, \mathrm{Mn}: 0.63 \%$ y Cr: $1.5 \%$. El material as-cast se homogenizó a $1000^{\circ} \mathrm{C}$ durante 6.5 horas tras lo cual se dejó enfriar en el horno.

Para establecer las temperaturas de austenización intercrítica se utilizaron las ecuaciones 1 y 2 [13]. Además, se determinaron las temperaturas de inicio y finalización de la austenita mediante dilatometría (Dilatómetro marca BAHR-DIL805A/D) a una velocidad de calentamiento de $0.2{ }^{\circ} \mathrm{C} / \mathrm{s}$.

$$
\begin{aligned}
& A_{c 1}=739-22.8 \mathrm{C}-6.8 \mathrm{Mn}+18.2 \mathrm{Si}+11.7 \mathrm{Cr}-15 \mathrm{Ni}-6.4 \mathrm{Mo}-5 \mathrm{~V}-28 \mathrm{Cu}(1) \\
& A_{c 3}=937.3-224.5 \sqrt{\mathrm{C}}-17 \mathrm{Mn}+34 \mathrm{Si}-14 \mathrm{Ni}+21.6 \mathrm{Mo}+41.8 \mathrm{~V}-20 \mathrm{Cu}(2)
\end{aligned}
$$

Dentro del intervalo de austenización intercrítica se escogieron dos temperaturas y se austenizaron muestras durante 15, 30 y 60 minutos, seguido de un enfriamiento rápido en agua. Las muestras obtenidas se prepararon mediante técnicas metalográficas tradicionales finalizando con alúmina de $0.3 \mu \mathrm{m}$ y ataque con Nital al 2\%. La fracción volumétrica de martensita en cada muestra se determinó bajo los lineamientos de la norma ASTM E562 mediante el método de conteo de puntos [14]. Para este propósito se analizaron 12 campos de visión hasta lograr una exactitud relativa menor al 10\%. La información obtenida sirvió para determinar las condiciones de austenización intercrítica. 
Posteriormente, se austenizaron intercríticamente muestras del acero bajo las condiciones determinadas en la etapa anterior, tras lo cual se austemperaron en un baño de sales tipo $\mathrm{KNO} 3-\mathrm{NaNO}_{2}$ a $350^{\circ} \mathrm{C}$ por 48 horas. En las muestras obtenidas se analizó la microestructura mediante microscopia óptica, microscopia electrónica de barrido y difracción de rayos $\mathrm{X}$.

La transformación de la austenita retenida a martensita bajo condiciones de deformación se estudió en muestras que se laminaron en frío a porcentajes de reducción de espesor de 5\%, 15\%, 20\% y 40\%. Antes y después de la laminación se midieron el largo $(l)$, el ancho $(w)$ y el espesor $(t)$ de las muestras y se calcularon las deformaciones reales y la deformación real total utilizando las ecuaciones 3 y 4 [1].

$$
\begin{aligned}
& \varepsilon_{\mathrm{t}}=\ln \left(\mathrm{t}_{\mathrm{l}} / \mathrm{t}_{0}\right), \varepsilon_{\mathrm{l}}=\ln \left(\mathrm{l}_{\mathrm{l}} / \mathrm{l}_{0}\right), \varepsilon_{\mathrm{w}}=\ln \left(\mathrm{w}_{\mathrm{l}} / \mathrm{w}_{0}\right) \\
& \varepsilon=\frac{\sqrt{2}}{3} \sqrt{\left(\varepsilon_{t}-\varepsilon_{l}\right)^{2}+\left(\varepsilon_{t}-\varepsilon_{w}\right)^{2}+\left(\varepsilon_{l}-\varepsilon_{w}\right)^{2}}
\end{aligned}
$$

En donde el subíndice 1 indica la dimensión de la muestra deformada y el subíndice 0 indica la dimensión de la muestra sin deformar, $\varepsilon_{i}$ es la deformación real en cada dirección $(l, w$ y $t)$ y $\varepsilon$ es la deformación real total.

La fracción volumétrica de austenita retenida se determinó mediante difracción de rayos X en las muestras laminadas, lo cual permitió determinar la variación en el contenido de austenita retenida en función de la deformación. Las mediciones por difracción de rayos $\mathrm{X}$ se realizaron en un difractómetro Panalytical Empyrean, entre $20^{\circ}-90^{\circ}$ con un paso de $0.013^{\circ}$ y un tiempo por paso de 50 s con radiación de cobre. Los difractogramas se analizaron con el programa X'pertHighScore Plus, versión 3.0.

\section{RESULTADOS}

La figura 1(a) muestra una curva de dilatometría representativa del acero homogenizado a la velocidad de calentamiento evaluada, en la que también se observa la primera derivada de la deformación con respecto a la temperatura. Las inflexiones en la primera derivada son las que permiten determinar las temperaturas de inicio y finalización de la austenita $\mathrm{A}_{\mathrm{C} 1}$ y $\mathrm{A}_{\mathrm{C} 3}$, las cuales dieron como resultado $786^{\circ} \mathrm{C}$ y $883^{\circ} \mathrm{C}$ respectivamente. Estos resultados fueron muy cercanos a los obtenidos utilizando las ecuaciones 1 y 2 tal como puede observarse en la tabla 1, a pesar de que los valores calculados se consideran para condiciones de equilibrio.

De acuerdo a estos resultados se seleccionaron las temperaturas de $790^{\circ} \mathrm{C} \mathrm{y} 800^{\circ} \mathrm{C}$ como temperaturas de austenización intercrítica y se sometieron muestras a tiempos de 15, 30 y 60 minutos, luego se hizo la cuantificación de la cantidad de martensita por conteo de puntos. Se asumió que la fracción volumétrica de martensita era la misma que la de la austenita de alta temperatura dado el carácter adifusional de la transformación. Los resultados mostraron, como era de esperarse, que la fracción volumétrica de martensita (austenita de alta temperatura) aumentaba al aumentar el tiempo y la temperatura de la austenización. Este comportamiento es característico durante la formación de la austenita en calentamiento, la cual involucra eventos de nucleación y crecimiento [15], [16]. Otros autores tales como SPEICH et al [17], han descrito las diferentes etapas de formación de la austenita en el rango intercrítico para aceros de bajo carbono con una microestructura inicial ferrítico-perlítica, en dónde la primera etapa de formación de la austenita es determinada por la disolución de la perlita y la velocidad de crecimiento es definida principalmente por la difusión del carbono en la austenita, con una distancia de difusión aproximadamente igual al espaciamiento interlaminar de la perlita. La etapa posterior de la formación de la austenita es a partir de la ferrita, y está regida principalmente por la difusión de los elementos sustitucionales tales como el silicio y el manganeso. La figura 2 muestra los resultados obtenidos. 

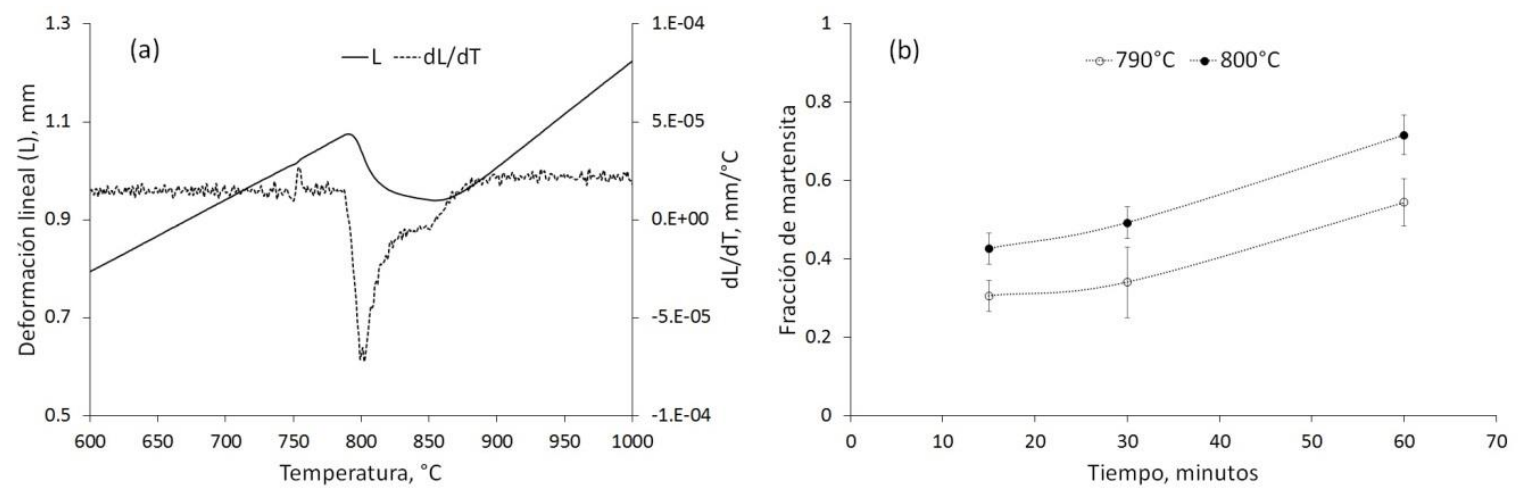

Figura 1:(a) Curva de dilatometría. (b) Fracción volumétrica de martensita en función de la temperatura y el tiempo de austenización intercrítica.

Tabla 1: Temperatura $\mathrm{A}_{\mathrm{C} 1}$ y $\mathrm{A}_{\mathrm{C} 3}$ para la muestra 1.

\begin{tabular}{c|c|c}
\hline$\mu$ & $\operatorname{Ac}_{1}\left({ }^{\circ} \mathrm{C}\right)$ & $\operatorname{Ac}_{3}\left({ }^{\circ} \mathrm{C}\right)$ \\
\hline Calculada & 781 & 879 \\
\hline Dilatometría & 786 & 883
\end{tabular}

La figura 2 muestra micrografías representativas de las muestras austenizadas intercríticamente y luego templadas. En las muestras austenizadas por 15 minutos se observó perlita sin disolver. Al aumentar el tiempo de austenización, se observó menor cantidad de perlita (P) sin disolver y un aumento en la fracción de martensita (M). Los granos de martensita muestran brazos creciendo hacia al interior de los granos ferríticos (F), lo cual sucede porque la fracción volumétrica de austenita de alta temperatura es mayor que la fracción volumétrica de perlita del material homogenizado, y por lo tanto la austenita crece inicialmente a expensas de la disolución de la perlita y luego por el consumo de los granos ferríticos. Luego de 1 hora de austenización intercrítica no se observó perlita sin disolver a ninguna de las dos temperaturas evaluadas.
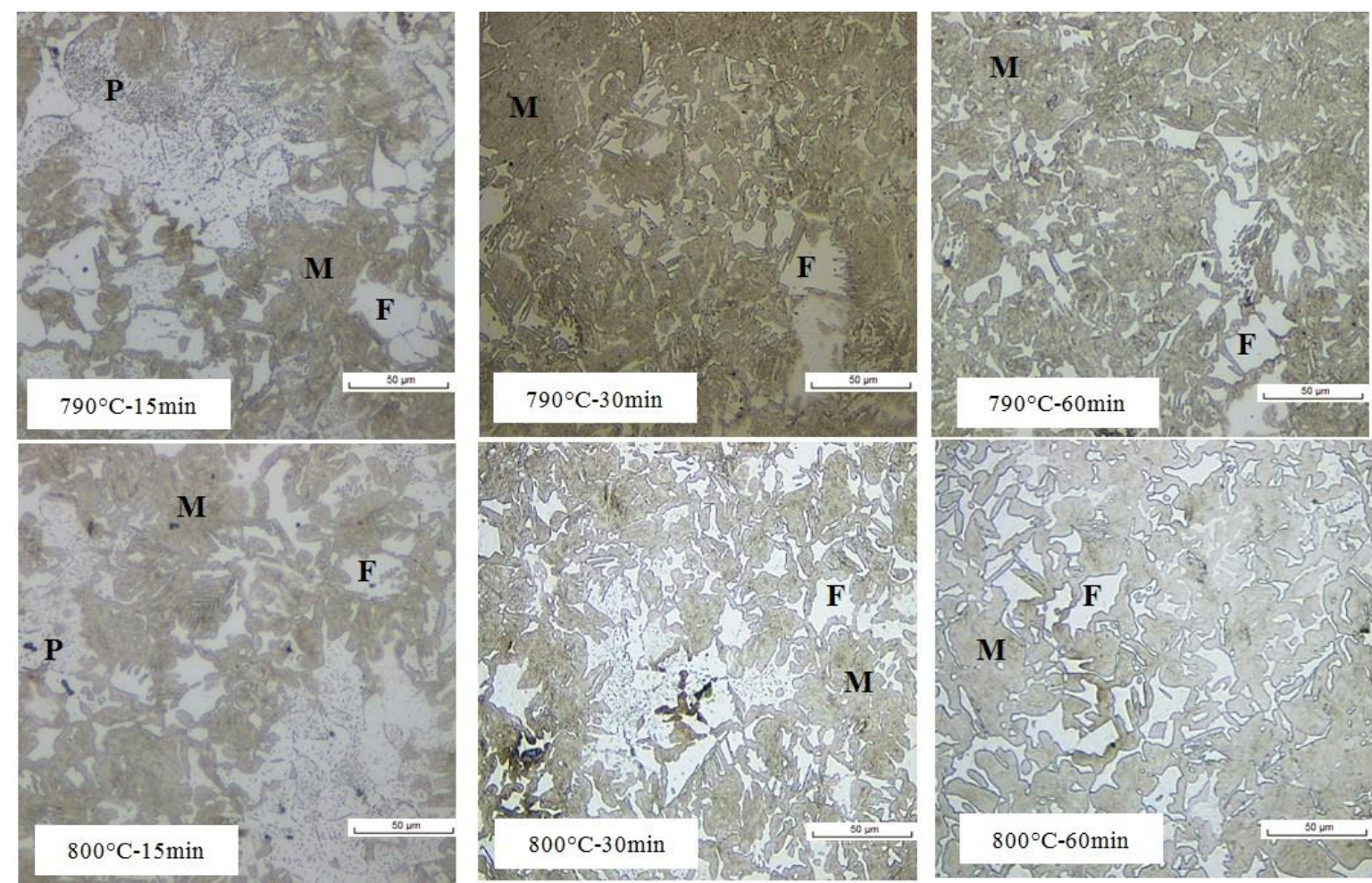

Figura 2: Micrografías representativas de los resultados de la austenización intercrítica. 
De acuerdo con los resultados de la etapa anterior, se seleccionaron temperaturas de $790^{\circ} \mathrm{C} \mathrm{y} 800^{\circ} \mathrm{C}$ y sostenimiento de una hora para la etapa de austenización intercrítica. Bajo estas condiciones las fracciones volumétricas de austenita a alta temperatura fueron de 0.54 y 0.71 respectivamente. Dado que la solubilidad del carbono en la ferrita es muy baja, se asumió que todo el carbono se difunde hacia la austenita, por lo tanto, los porcentajes de carbono en la austenita para cada acero corresponden a $0.46 \%$ para el acero austenizado intercríticamente a $790^{\circ} \mathrm{C}-1 \mathrm{~h}$ y $0.35 \%$ para el acero austenizado a $800^{\circ} \mathrm{C}-1 \mathrm{~h}$. La ecuación 5 permite calcular la temperatura de inicio de la martensita de acuerdo a la composición química [18]. Debido a que la muestra antes de la austenización intercrítica se encuentra en estado homogenizado y a que la velocidad de difusión de los elementos sustitucionales a las temperaturas de trabajo es baja, se asumió que el contenido de elementos de aleación en la austenita de alta temperatura es igual al del metal base. Cabe anotar que es de esperarse una leve partición de los elementos de aleación, por ejemplo, el silicio y el cromo deberían particionarse preferencialmente hacia la ferrita, mientras el manganeso debería particionarse hacía la austenita. Los resultados indicaron una temperatura de inicio de la martensita de $286^{\circ} \mathrm{C}$ y $332^{\circ} \mathrm{C}$ para las muestras austenizadas intercríticamente a $790^{\circ} \mathrm{C}$ y $800^{\circ} \mathrm{C}$ respectivamente, razón por la cual se decidió realizar el austemperado a $350^{\circ} \mathrm{C}$, el tiempo de austemperado de 48 horas se seleccionó basado en reportes de otros investigadores que han utilizado aleaciones con composiciones químicas similares a la de la austenita de alta temperatura de este estudio para obtener aceros nanobainíticos [19], [20].

$$
M_{S}=539-423 \mathrm{C}-30,4 \mathrm{Mn}-17,7 \mathrm{Ni}-12,1 \mathrm{Cr}-11,0 \mathrm{Si}-7,07 \mathrm{Mo}
$$

La figura 3 muestra micrografías representativas de las muestras austenizadas intercríticamente y luego austemperadas. En las figuras 3(a) y 3(c) adquiridas con microscopia óptica, se observa la presencia de ferrita proeutectoide (FP), bainita (B) y martensita (M). Las figuras 3(b) y 3(d) muestran micrografías representativas de las zonas bainíticas obtenidas en el microscopio electrónico de barrido, en donde se observa austenita retenida y ferrita bainítica (FB). La austenita retenida incluye austenita en bloque (AB) con tamaños cercanos a $1 \mu \mathrm{m}$ y austenita en placas (AP) con espesores que van hasta los $200 \mathrm{~nm}$.
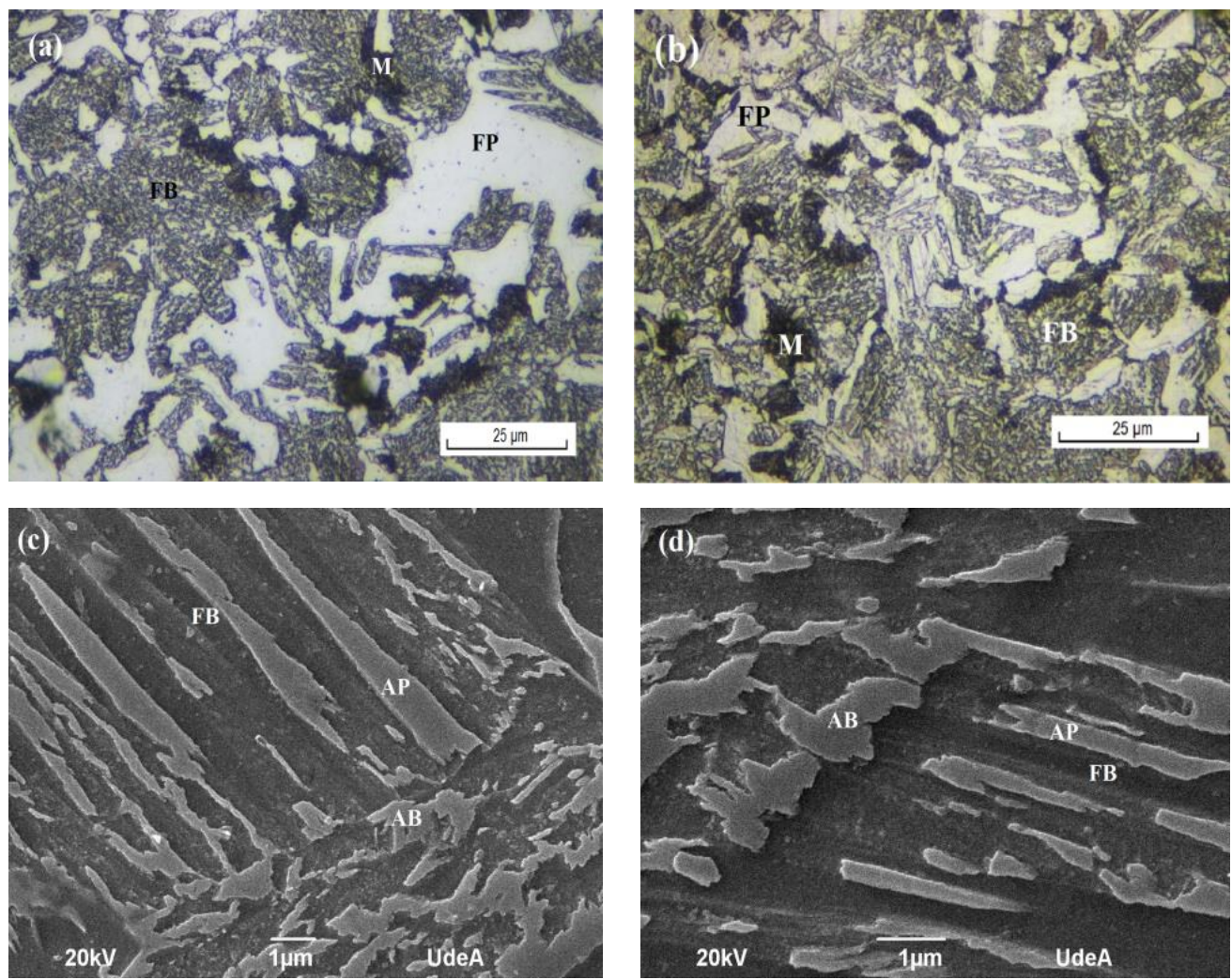

Figura 3: Micrografías de los materiales austenizados intercríticamente a: (a) y (c) $790^{\circ} \mathrm{C}-1 \mathrm{~h}$, (b) y (d) $800^{\circ} \mathrm{C}-1 \mathrm{~h}$, mediante microscopia óptica y microscopia electrónica de barrido, respectivamente. Todos los materiales fueron austemperados a $350^{\circ} \mathrm{C}$ durante 48 horas. 
Mediante difracción de rayos X (figura 4) se determinaron las fracciones volumétricas de austenita retenida en cada material dando como resultado $5.9 \%$ vol y $3.3 \%$ vol para las muestras austenizadas intercríticamente a $790^{\circ} \mathrm{C}$ y $800^{\circ} \mathrm{C}$, respectivamente. La ferrita proeutectoide en la microestructura corresponde a la fracción del material sin austenizar durante la austenización intercrítica. La martensita se forma a partir de la austenita de alta temperatura que no alcanzó a transformase durante el tratamiento isotérmico y que tampoco alcanzó a enriquecerse lo suficiente en carbono para ser estable a temperatura ambiente.

Finalmente, la bainita es producto de la transformación isotérmica, el pequeño tamaño de las placas de ferrita bainítica y austenita retenida se debe a la baja temperatura de la transformación. Además, la presencia de austenita retenida en lugar de carburos se atribuye a la alta concentración de silicio en la aleación, ya que el silicio retarda la precipitación de los carburos durante la reacción bainítica, promoviendo la presencia de austenita retenida rica en carbono y estable a temperatura ambiente.

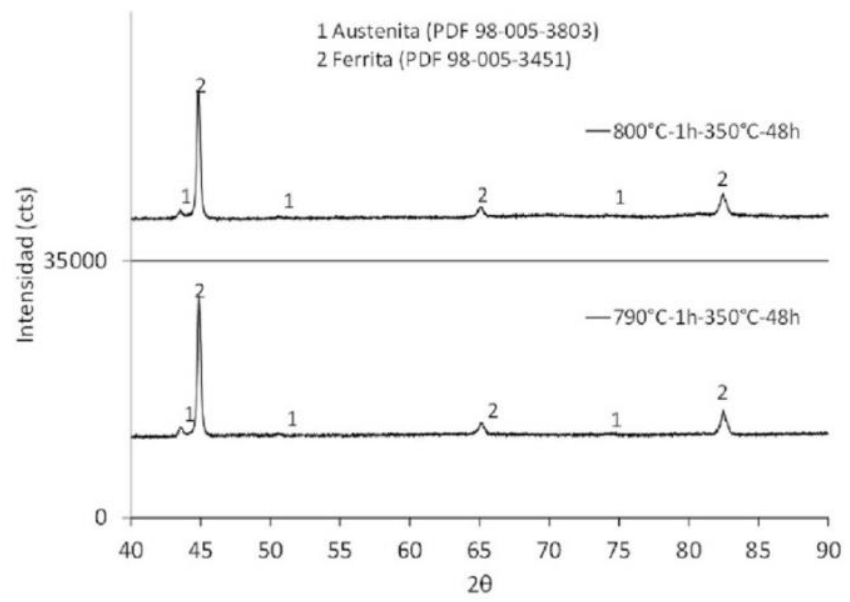

Figura 4: Difractogramas de los aceros austenizados intercríticamente y austemperados.

La figura 5 muestra la variación de la fracción de austenita transformada en función de la deformación y de la temperatura de austenización intercrítica. El análisis de la transformación de la austenita a martensita bajo condiciones de deformación mostró que alrededor del 50\% de la austenita retenida se transformó a martensita antes de alcanzar deformaciones reales totales del 10\%. A deformaciones mayores la tasa de transformación disminuyó hasta mostrar una tendencia a detenerse cerca del 30\% de deformación. La muestra austenizada a $790^{\circ} \mathrm{C}$ presentó una velocidad de transformación mayor y alcanzó mayores porcentajes de transformación que la austenizada a $800^{\circ} \mathrm{C}$, indicio de que la muestra austenizada a mayor temperatura presentó una austenita más estable. El comportamiento de la fracción de austenita retenida transformada con respecto a las deformaciones reales, obtenido para los aceros de estudio, es similar al obtenido por GARCÍA-MATEO et al [21] para aceros Nanobain austenizados completamente, con contenidos de C, Si y Cr similares a los de los aceros de estudio, pero con mayores de Mn (2\%) y Co $(1.5 \%)$ y los cuáles fueron conformados mediante laminación en caliente.

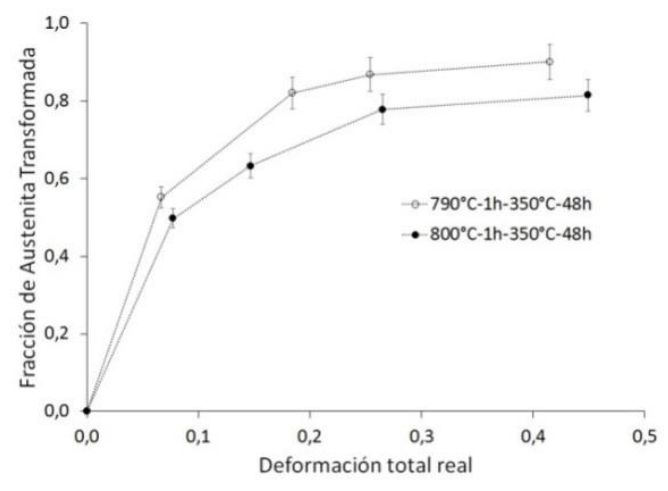

Figura 5: Fracción de austenita transformada en función de la temperatura. 
La estabilidad de la austenita retenida está relacionada con su composición química en especial con la concentración de carbono y con factores como el tamaño, morfología y propiedades de las fases alrededor de la austenita. Con el fin de establecer más exactamente las causas asociadas a la diferencia entre las aleaciones obtenidas, se requiere una caracterización más profunda.

\section{CONCLUSIONES}

Mediante austenización intercrítica seguida de austemperado se obtuvieron aceros multifásicos con ferrita proeutectoide, martensita y bainita libre de carburos con austenita en bloque y en láminas, esta última de tamaño nanométrico. La evaluación de la estabilidad de la austenita bajo condiciones de deformación indicó que al aumentar la temperatura de austenización intercrítica se disminuye la fracción de austenita retenida en la microestructura y se obtiene una austenita más estable. Se requiere una caracterización microestructural más profunda para identificar las causas de este comportamiento, que pueden incluir diferencias en la composición química, tamaño y morfología de la austenita. También son necesarios ensayos para determinar las propiedades mecánicas de las aleaciones obtenidas. La combinación de austenización intercrítica seguida de austemperado a baja temperatura de una aleación con la composición química adecuada, permitió obtener aceros que combinan la microestructura de los aceros TRIP y NANOBAIN.

\section{AGRADECIMIENTOS}

Los autores agradecemos al CODI de la Universidad de Antioquia por la financiación a través del proyecto PRG2014-1038, de la estrategia de sostenibilidad 2014-2015 y de la Beca Joven Investigador. Al laboratorio de Fundición de la Universidad de Antioquia y al laboratorio de Materialografía del Instituto Tecnológico Pascual Bravo.

\section{BIBLIOGRAPHY}

[1] TIMOKHINA, I.B., HODGSON, P.D., PERELOMA, E.V., "Effect of microstructure on the stability of retained austenite in transformation-induced-plasticity steels", Metallurgical and Materials Transactions A, v. 35A, n. 8, pp. 2331-2341, 2004.

[2] ZAEFFERER, S., OHLERT, J., BLECK, W., "A study of microstructure, transformation mechanisms and correlation between microstructure and mechanical properties of a low alloyed TRIP steel", Acta Materialia, v. 52A, n. 9, pp. 2765-2778, 2004.

[3] WORLD STEEL ASSOCIATION, http://www.worldautosteel.org/steel-basics/steeltypes/transformation-induced-plasticity-trip-steel/ Fecha de consulta: 9 de noviembre de 2017.

[4] CHRISTODOULOU, P.I., KERMANIDIS, A.T., KRIZAN, D., "Fatigue behavior and retained austenite transformation of Al-containing TRIP steels", International Journal of Fatigue, v. 91, pp. 220-231, 2016.

[5] COVARRUBIAS ALVARADO, O. "Efectos microestructurales en un acero sometido a un tratamiento térmico TRIP," Tesis de Doctor en ciencias de la ingeniería mecánica con especialidad en materiales, Universidad Autónoma de Nuevo León, México, 2002.

[6] HOSSEINI, S.M.K., ZAREI-HANZAKI, A., YUE, S. "Effects of ferrite phase characteristics on microstructure and mechanical properties of thermomechanically-processed low-silicon content TRIP-assisted steels", Materials Science \& Engineering A, v. 626, pp. 229-236, 2015.

[7] CABALLERO, F.G., GARCÍA-MATEO, C., BHADESHIA, H.K.D.H., “Acero NANOBAIN: Un material del siglo XXI", Sociedad Española de Materiales, pp. 1-2, 2008.

[8] MORALES-RIVAS, L., GARCÍA-MATEO, C., KUNTZ, M., et al., "Induced martensitic transformation during tensile test in nanostructured bainitic steels," Materials Science \& Engineering A, v. 662, pp. 169-177, 2016.

[9] SHERIF, M.Y., GARCÍA-MATEO, C., SOURMAIL, T., et al., "Stability of retained austenite in TRIPassisted steels", Material Science Technology, v. 20, n. 3, pp. 319-322, 2004.

[10] CHIANG, J., LAWRENCE, B., BOYD, J.D., et al., "Effect of microstructure on retained austenite stability and work hardening of TRIP steels", Materials Science and Engineering A, v. 528, n. 13-14, pp. 45164521, 2011.

[11] REISNER, G., WERNER, E.A., KERSCHBAUMMAYR, P., et al., "The modeling of retained austenite in low-alloyed TRIP steels", JOM, v. 49, n 9, pp. 62-65, 1997. 
[12] GARCÍA-MATEO, C., CABALLERO, F.G., "The Role of Retained Austenite on Tensile Properties of Steels with Bainitic Microstructures,” Materials Transactions, v. 46, n. 8, pp. 1839-1846, 2005.

[13] TRZASKA, J., DOBRZANSKI, V., "Modelling of CCT diagrams for engineering and constructional steels", Journal of Materials Processing Technology, v.192-193, pp. 504-510, 2007.

[14] ASTM, "Designation E562 - 11, Standard Test Method for Determining Volume Fraction by Systematic Manual Point Count," Practice, pp. 1-7, 2011.

[15] CHRISTIAN, J.W., "The formation of austenite from two-phase mixtures of ferrite and cementite," En: The theory of transformations in Metals amd Alloys, Part II, Elservier Science LLtd, pp. 813-817, 2002.

[16] HUANG, J., POOLE, W.J., MILITZER, M., “Austenite formation during intercritical annealing”, Metallurgical and Materials Transactions A, v.35, n. 11, pp. 3363-3375, 2004.

[17] SPEICH, G. R., DEMAREST, V.A., MILLER, R.L., "Formation of Austenite During Intercritical Annealing of Dual-Phase Steels," Materials Transactions A, v. 12, n. 8, pp. 1419-1428, 1981.

[18] ANDREWS, K., "Empirical Formulae for the Calculation of Some Transformation Temperatures", Journal of the Iron Steel Institute., v. 203, n. 7, pp. 721-727, 1965.

[19] HAN, Y., WU, H., LIU, C., "Microstructures and Mechanical Characteristics of a Medium Carbon Super-Bainitic Steel After Isothermal Transformation", Journal of Materials Engineering and Performance, v. 23, n. 12, pp. 4230-4236, 2014.

[20] WASILUK, K., WASIAK, K., SKOŁEK, E., ŚWIĄTNICKI, W., "Formation of nanobainitic structure in carburized layer of structural steels", Metal, pp. 1-7, 2014.

[21] GARCIA-MATEO, C., CABAllerO, F. G., CHAO, J., CAPDEVILA, C., GARCIA DE ANDRES, C., "Mechanical stability of retained austenite during plastic deformation of super high strength carbide free bainitic steels," Journal of Materials Science, v. 44, n. 17, pp. 4617-4624, 2009. 